\title{
Application of New Qualitative Voicing Time-Frequency Features for Speaker Recognition
}

\author{
Nidhal Ben Aloui ${ }^{1}, 2$, Hervé Glotin ${ }^{1}$, and Patrick Hebrard ${ }^{2}$ \\ ${ }^{1}$ Université du Sud Toulon-Var Laboratoire LSIS \\ B.P. 20132 - 83957 La Garde, France \\ \{benaloui,glotin\}@univ-tln.fr \\ 2 DCNS - Division SIS, \\ Le Mourillon B.P. 403 - 83055 Toulon, France \\ \{nidhal.ben-aloui, patrick.hebrard\}@dcn.fr
}

\begin{abstract}
This paper presents original and efficient Qualitative TimeFrequency (QTF) speech features for speaker recognition based on a med-term speech dynamics qualitative representation. For each frame of around $150 \mathrm{~ms}$, we estimate and binarize a suband voicing activity estimation of 6 frequency subands. We then derive the Allen temporal relations graph between these 6 time intervals. This set of temporal relations, estimated at each frame, feeds a neural network which is trained for speaker recognition. Experiments are conducted on fifty speakers (males and females) of a reference radio database ESTER (40 hours) with continuous speech. Our best model generates around 3\% of frame class error, without using information of frame continuity, which is similar to state of the art. Moreover, our QTF generates a simple and light representation using only 15 integers for coding speaker identity.
\end{abstract}

\section{Introduction}

Classical models of speech recognition assume that a short term analysis of the acoustic signal is essential for accurately decoding the speech signal. Most of these systems are based on maximum likelyhood trained Gaussian mixture speaker models, with diagonal covariance matrices. They can use for example principal component analysis from the output of 24 mel frequency channels, or more usually around 256 components calculated from the cepstral coefficients and deltas [1], voice recognition feature for speaker recognition such as since such features compensate charactarics of speakers [2]

This paper presents an alternative view, where the time scale requires an accurate description, longer than the phonetic segment, wedded to the dynamics of TF voicing level intervals. We assume that voicing reflects a singular property of the modulation spectrum that may provide a qualitative framework to generate a speaker identity model. 
A variety of studies have shown that intelligibility depends on the integrity of the low-frequency modulation spectrum [3. Within reverberant environments the greatest impact of acoustic reflection is between 2 and $6 \mathrm{~Hz}$. An effect of reverberation is to jumble the spectral content of the acoustic signal across both time and frequency, particulary that portion of the spectrum below $1500 \mathrm{~Hz}$. Although, reverberation is known to interfere with intelligibility; the basis for its deleterious impact is not well understood. On the other hand, it has been established that phonological perception is a SB process [4. Moreover, this can be linked to speech production dynamics 5. This has inspired various algorithms for robust speech recognition [6, 7, also linked to the TF voicing level [6, 8, This time-frequency dynamics seems therefore to be important in speech perception, and because a natural representation for timely events has been proposed by Allen J.F., we proposed a quantic time-frequency dynamics for robust speech features extraction paradigm [9], that an approach is presented here for a specific task of speaker recognition.

\section{Features Extraction}

\subsection{Voicing Extraction}

We use the voicing measure [6, 10, correlated with SNR and equivalent to the harmonicity index (HNR) 11, 12 to estimate the average voicing per utterance. It is extracted form the autocorrelogram of a demodulated signal. In the case of Gaussian noise, the correlogram of a noisy frame is less modulated than a clean one 11. The peaks in the autocorrelogram of the demodulated frame isolate the various harmonics in a signal. This can be used to separate a mixture of harmonic noises and a dominant harmonic signal. It is interesting that such separation can be efficiently accomplished, using a time windows of duration in the same range as the average phoneme duration.

Before the autocorrelation we compute the demodulated signal after half wave rectification, followed by pass-band filtering in the pitch domain $([90,350] \mathrm{Hz})$. For each frame of $128 \mathrm{~ms}$, we calculate the ratio $R=R 1 / R 0$, where $R 1$ is the local maximum in time delay segment corresponding to the fundamental frequency, and $R 0$ is the cell energy. This measure is strongly correlated with SNR in the 5-20dB range [1]. Figure 1 demonstrates explicitly the voicing levels for each SBs and each frame. These values will be thresholded to get qualitative dynamic features that will be used to estimate the speaker identity. The subband (SB) definitions are following Fletcher studies, followed by other ones like in ALLEN J.B. papers 4, 5, 6, 1. Here are the definitions of SB range $(\mathrm{Hz}):[216778 ; 7071631 ; 12622709 ; 21213800 ; 34005400 ; 5000$ 8000].

${ }^{1}$ Note that ALLEN J.B and ALLEN J.F. are two different authors, the first worked on speech analysis, the second on generic time representation. Our model is based on both approaches. 


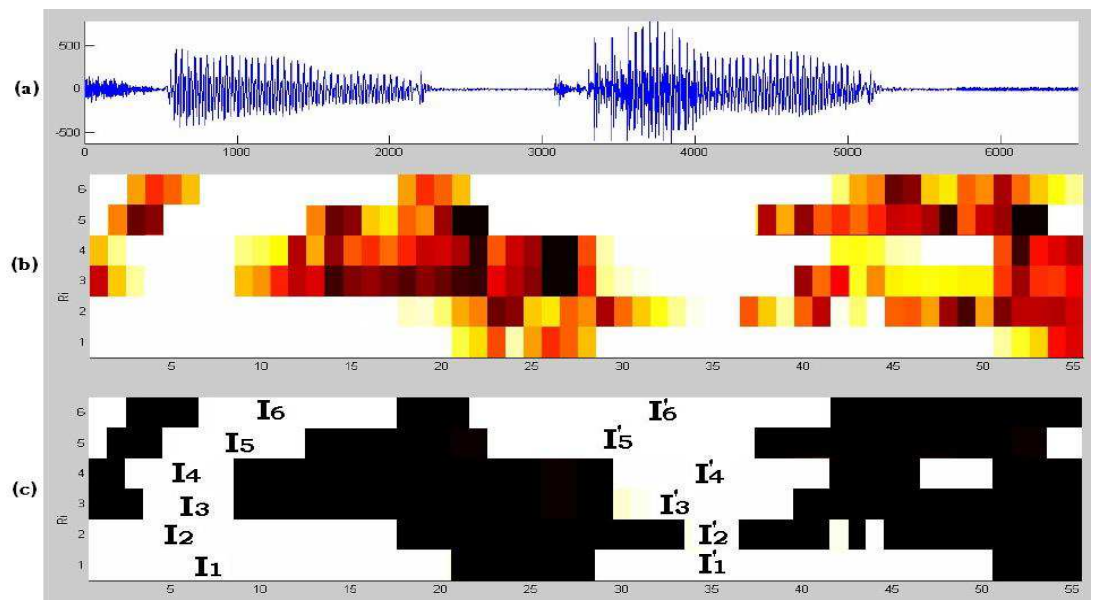

Fig. 1. From Voicing to the Allen's Interval : (a) voicing signal (b) the voicing level by $\mathrm{SB}(\mathrm{c})$ the binarized voicing levels by $\mathrm{SB}$ using ${ }^{s} T_{b}$ threshold

\subsection{Allen Interval Graphs}

A temporal intervals algebra has been defined in [13, 14, where 13 atomic relations are depicted between two time intervals. From ours observations to the voicing levels by SB (cf fig. 1 1 (b)), we propose to apply the J.F. Allen's time representation for each SBs and each voicing activity regions (that can be 200 ms long). We present Allen's time relationships in the figure 2 $X$ is the sliding interval which gives, progressively with $Y$, the 13 Allen intervals relations. We can define the algebric distance $d$ between the two nearest interval to 1 and we increment it as we moved away from the interval relation. This will give us the numeric values for each relation that we use in this paper. The "b"symbol is coded into "1", "m"into "2", ... for the 13 relations of the figure 2, Moreover, the "no-relation" is also coded into "0", this can occured between two SBs if one or both have no frame with enough voicing level.

In order to obtain and to characterize exactly these six SB intervals we binarize the voicing matrix ${ }^{s} M_{R}$ (s mean that for each speaker we build his own voicing matrix). First of all, we must find the best threshold for each sub-waveband to use it after. Indeed, after some experiences we have noticed that it is better to set one threshold for each SB. Thus we are looking for the best threshold which gives a fixed percent $(30 \%, 50 \%$ and $70 \%)$ of 1 in each waveband by binarising the voicing matrix ${ }^{s} M_{R}$. We first look for the threshold ${ }^{s} T_{b_{i}}$ values in:

$\Upsilon=\left[\operatorname{mean}\left({ }^{s} M_{R_{b_{i}}}\right)-\tau\right.$, mean $\left.\left({ }^{s} M_{R_{b_{i}}}\right)+\tau\right]$, by steps of 0.01 with $\tau=0.4$, such that:

$$
\frac{\operatorname{Card}\left({ }^{s} R_{b_{i}}(t)>{ }^{s} T_{b_{i}}\right)}{\operatorname{Card}\left({ }^{s} w\right)}=P \pm \varepsilon,
$$




\begin{tabular}{|c|c|c|c|}
\hline Relation & Symbol & Illustration & Inverse Sy \\
\hline $\mathrm{X}$ before $\mathrm{Y}$ & b & $\stackrel{X}{Y}, Y$ & $\mathrm{a}$ \\
\hline $\mathrm{X}$ meets $\mathrm{Y}$ & $\mathrm{m}$ & $\stackrel{X}{X}$ & $\mathrm{mi}$ \\
\hline X overlaps Y & o & $\stackrel{X}{\longmapsto} Y$ & oi \\
\hline $\mathrm{X}$ starts Y & $\mathrm{s}$ & $\left.\right|_{\mathrm{Y}}$ & si \\
\hline $\mathrm{X}$ during $\mathrm{Y}$ & $\mathrm{d}$ & $\frac{{ }^{\mathrm{X}}}{\mathrm{Y}}$ & di \\
\hline$X$ finishes $Y$ & $\mathrm{f}$ & $\mathrm{Y}^{\longmapsto}$ & fi \\
\hline $\mathrm{X}$ equals $\mathrm{Y}$ & eq & $\frac{\mathrm{X}}{\mathrm{Y}}$ & \\
\hline
\end{tabular}

Fig. 2. The interval construction structure with the 13 symbols. ( no="no-relation").

where $\mathrm{t}$ is the time of speaker $\mathrm{s}, \mathrm{T}$ is threshold used, $b_{i}$ represents a $\mathrm{SB}$ with $i \in\{1 . .6\}$, and $\mathrm{P}$ defines the percent of 1 in the binarized interval. $\varepsilon$ is a percent of tolerance $( \pm 1 \%)$, and ${ }^{s} w$ is the total number of frames for speaker $s$.

If not any threshold value satisfies the previous conditions, then we set ${ }^{s} T_{b_{i}}=$ $\operatorname{mean}\left({ }^{s} M_{R_{b_{i}}}\right)$.

After this iterative threshold research, we have generated per speaker voicing frame a threshold vector ${ }^{s} T=\left[\begin{array}{llllll}{ }^{s} T_{1} & { }^{s} T_{2} & { }^{s} T_{3} & { }^{s} T_{4} & { }^{s} T_{5} & { }^{s} T_{6}\end{array}\right]$, where $T_{i}$ represents the threshold by SB. By applying ${ }^{s} T$ to ${ }^{s} M_{R}$ we obtain the binarized matrix, which a short sample is represented on Fig. 1 .c. We then extract the Allen's graph per speaker for all frame having at least 4 binarized voicing interval simultaneously equal to 1 .

The Allen graph corresponding to the figure 1 $\mathrm{fc}$ is represented by atomic relation matrix in figure 3 (we characterize in bold the relations which are non redundant and useful). For each speaker, we obtain full graphs which will be used to discriminate one from the other by using a Multi-Layer Perceptron (MLP). In the next paragraph, we introduce a brief explanation of this method.

All Allen graphs are symmetric to the first diagonal (see 3). Each of the seven relations has an inverse relation in the same graph. This is due to the logic between two intervals: if the interval $\mathrm{A}$ is BEFORE $\mathrm{B}$, in the same time we can assure that B IS AFTER A. Thus the useful information is contained in the 15 first relations which are ordered from left to the right, beginning from subband I'1 to subband I'5. For our example, one input vector at time t of our Multi Layer Perceptron Classifier is $\mathrm{QTF}(\mathrm{t})=[\mathrm{di}$ di di oi oi $\mathrm{d} d \mathrm{~d} d \mathrm{~s}$ oi $\mathrm{d}$ oi $\mathrm{f} d$ ]. We see on figure 4 the histogram of all QTF vectors for "Yves Decaens". 


\begin{tabular}{|c|c|c|c|c|c|}
\hline & $I_{1}^{\prime}$ & $I_{2}^{\prime}$ & $I_{3}^{\prime}$ & $I_{4}^{\prime}$ & $I_{5}^{\prime}$ \\
\hline$I_{1}^{\prime}$ & $e q$ & di & di & di & oi \\
\hline$I_{2}^{\prime}$ & $d$ & $e q$ & d & d & d \\
\hline$I_{3}^{\prime}$ & $d$ & $d i$ & $e q$ & $\mathbf{s}$ & oi \\
\hline$I_{4}^{\prime}$ & $d$ & $d i$ & si & $e q$ & oi \\
\hline$I_{5}^{\prime}$ & $O$ & $d i$ & $O$ & $O$ & $e q$ \\
\hline$I_{6}^{\prime}$ & $o$ & $d i$ & $d i$ & fi & $d i$ \\
\hline
\end{tabular}

Fig. 3. The second part of the figure 1c Allen graph correspondences. The top-right triangle of this matrix contains the 15 relations values that will be given to an MLP for Id. speaker modelisation. Then they will be sorted from 1 to 15 as $1=\left(I^{\prime} 1, I^{\prime} 2\right), 2=$ $\left(I^{\prime} 1, I^{\prime} 3\right), \ldots, 15=\left(I^{\prime} 5, I^{\prime} 6\right)$. We needn't to use down-left triangle of this matrix due to the symmetric relation between both triangles.

The "no-relation" occures when some binarized SBs only containing zeros. We see that that the "no-relation" represents around $10 \%$ of all relations. See also Tab. 1 for an estimation of the discrimative power of this relation.

In figure 5 we present the log ratio of the QTF normalized distributions of two speakers (Yves Deceans and Patricia Martin), whos both have spoken about 50 minutes. It shows well the strong differences for certain relations and certain SB couples. We demonstrate in this paper that this information is discrimitive for a speaker identification task training a simple MLP.

For this purpose, we train with these 15 integers a MLP using the Torch toolbox 15. It's a machine learning library written in simple $\mathrm{C}++$ and distributed under BSD License. Torch is currently developed by IDIAP team. Torch has been designed to be essentially time efficient, modular, as it is a research oriented library. Our MLP is based on the Last Mean Square criterion (other Machine Learning algorithm could have been used, like Support Vector Machines SVM).

\section{Database}

Our experiments are conducted on a corpus issued from the Phase 1 of ESTER 2006 evaluation campaign (Evaluation Campaign for the Rich Transcription of French Broadcast News) [16. ESTER implements three tasks, named transcription $(\mathrm{T})$, segmentation $(\mathrm{S})$ and information extraction $(\mathrm{E})$. We have focused our work on SVL Speaker tracking and SRL Speaker diarization. This acoustic corpus Phase 1 is issued from two different sources, named France Inter (Inter) and Radio France International (RFI). It contains about 40 hours of manually transcribed radio broadcast news. This corpus is divided into three separated parts for training, developing and testing activities respectively. The training part (train) contains 30 hours and 40 minutes and the development part (dev) 4 hours and 40 minutes. The test part (test) contains 4 hours and 40 minutes. The unseen source in the test data is meant to evaluate the impact of the knowledge of the document source on performances. 


\section{Experimental Results and Interpretation}

We give class error rate results left column in table 1 and their falsification in right columns in the same Table. We see that the best parametrisation is given for $70 \%$ of 'active'intervall in each SB, yielding to a class error rate of $2.5 \%$. Comparison with ESTER Phase 1 results [17] are promising: we get the same order of speaker identification for this task on continous broadcast news.

Table 1. In the "Real Data" column are presented the class error results for the 50 speakers id. with different $P$ values (MLP methods $P=0.3,0.5$ or 0.7 ). The best score is around $2.5 \%$. In the "Falsified Data" column we find for comparison a model falsification with only two relations: no-relation versus all other relations (see text).

\begin{tabular}{|c|cc|cccc||ccc||c|}
\hline & & & \multicolumn{4}{|c||}{ Real data } & \multicolumn{4}{c|}{ Falsified data } \\
\hline \multirow{3}{*}{$\begin{array}{c}\text { MLP } \\
\text { method }\end{array}$} & Parameters & \multicolumn{4}{|c||}{ The_class_error } & \multicolumn{4}{c|}{ The_class_error } \\
data Set & $\mathrm{nhu}$ & iter & Train & Dev & Test & iter & Train & Dev & Test \\
$\mathbf{0 . 3}$ & - & 300 & 1206 & 8.57 & 12.37 & 12.21 & 132 & 85.21 & 85.01 & 84.95 \\
& 27338 & 600 & 1678 & 9.97 & 16.18 & 16.27 & 123 & 85.15 & 84.44 & 85.29 \\
& - & $\mathbf{6 0 0}$ & $\mathbf{1 4 7 2}$ & $\mathbf{8 . 2 0}$ & $\mathbf{1 3 . 6 2}$ & $\mathbf{1 3 . 3 0}$ & 110 & 85.14 & 84.21 & 84.50 \\
& - & 1000 & 1242 & 26.16 & 32.93 & 33.68 & $\mathbf{1 3 8}$ & $\mathbf{8 4 . 9 9}$ & $\mathbf{8 5 . 7 4}$ & $\mathbf{8 6 . 1 2}$ \\
\hline \multirow{3}{*}{$\mathbf{0 . 5}$} & - & 300 & 1431 & 8.58 & 13.32 & 13.11 & $\mathbf{9 5}$ & $\mathbf{8 5 . 2 2}$ & $\mathbf{8 4 . 6 0}$ & $\mathbf{8 4 . 9 6}$ \\
& 27306 & $\mathbf{6 0 0}$ & $\mathbf{1 9 4 0}$ & $\mathbf{6 . 6 2}$ & $\mathbf{9 . 0 5}$ & $\mathbf{9 . 1 1}$ & 140 & 85.23 & 85.33 & 84.80 \\
& - & 600 & 675 & 34.95 & 43.22 & 42.58 & 128 & 85.24 & 84.86 & 84.57 \\
& - & 1000 & 1560 & 10.20 & 15.17 & 15.11 & 130 & 85.07 & 85.24 & 85.70 \\
\hline \multirow{3}{*}{$\mathbf{0 . 7}$} & 18166 & 600 & 874 & 1.56 & 2.68 & 3.01 & 159 & 86.03 & 86.74 & 86.03 \\
& - & 300 & 819 & 1.49 & 2.7 & 2.92 & 141 & 85.75 & 86.56 & 85.90 \\
& - & $\mathbf{6 0 0}$ & $\mathbf{8 7 3}$ & $\mathbf{1 . 4 4}$ & $\mathbf{2 . 5 5}$ & $\mathbf{2 . 4 6}$ & $\mathbf{1 4 9}$ & $\mathbf{8 6 . 1 1}$ & $\mathbf{8 6 . 4 1}$ & $\mathbf{8 6 . 2 5}$ \\
& - & 1000 & 923 & 1.46 & 2.57 & 2.48 & 150 & 86.11 & 86.92 & 86.01 \\
\hline
\end{tabular}

We run a falsification experimentation to verify if each relation encodes useful information, or if only the difference between "no-relation" and all other relations is relevant. Thus, in each set (train, dev and test), we have just used a 1 bit intput parameter for our MLP, replacing all atomic relations different to the "no-relation" by 1 , and keeping all the no-relation (originaly coded by 0 ). The table 1 on the right gives this falsification results: we observe that the worst score is approximately $85 \%$, telling that most of the coding information is given by the nature of the relations. But we also mesure that this is better than the random system (around 93\% cf. formuld 2). This demonstrates that the norelation contains by itself some speaker identity information.

${ }^{2}$ Let $P_{k}$ be the frequency of the class $C_{k}$, the error rate of a random classifier is:

$$
E R_{\text {rand }}=1-\sum_{k=1}^{c}\left(P_{k}\right)^{2}=1-\sum_{k=1}^{c}\left(\frac{\operatorname{card}\left(C_{k}\right)}{\sum_{k=1}^{c} \operatorname{card}(C)}\right)^{2}
$$

where $c$ is the number of classes and $\operatorname{card}\left(C_{k}\right)$ is the number of images in the class $C_{k}$. 


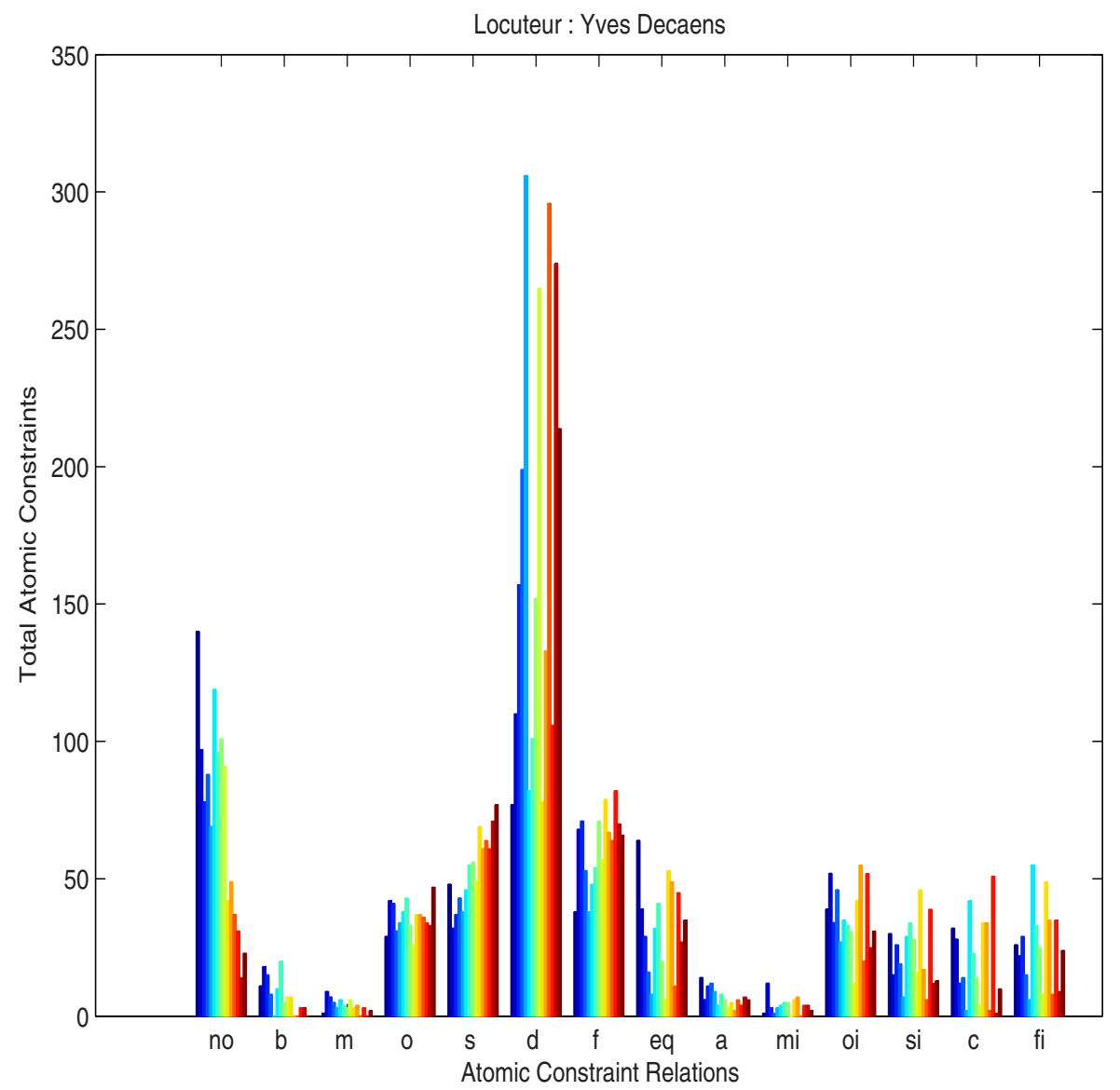

Fig. 4. Histogram of the QTF MLP inputs for the men speaker "Yves DECAENS". Each atomic relation is in abscissa from left to right (no:no-relation, b:before, m:meets, o:overlaps, s:starts, d:during, f:finishes, eq:equals, and respective symmetric ones: a, mi, oi, si, di, fi and eq). Each of the 15 MLP inputs (from left blue for the SB couple (I1,I2), to right red dark for the SB couple (I5,I6)). We note that the QTF distributions are varying more across relations than across each SB couples. The first input in dark blue on the left of each histogram (i.e for the couple (I1,I2)) has a majority of "no relation" and "during". The 15th input (node for couple (I5,I6)), in the right of each histogram, has a majority of "during" relation, ie the voicing interval in subband 5 is most of the time included in the voicing interval of SB 6. The "meeting" relations are not present for this speaker. The "equal" relation seems to be an informative kind of relation, showing great contrast across each SB couple. 


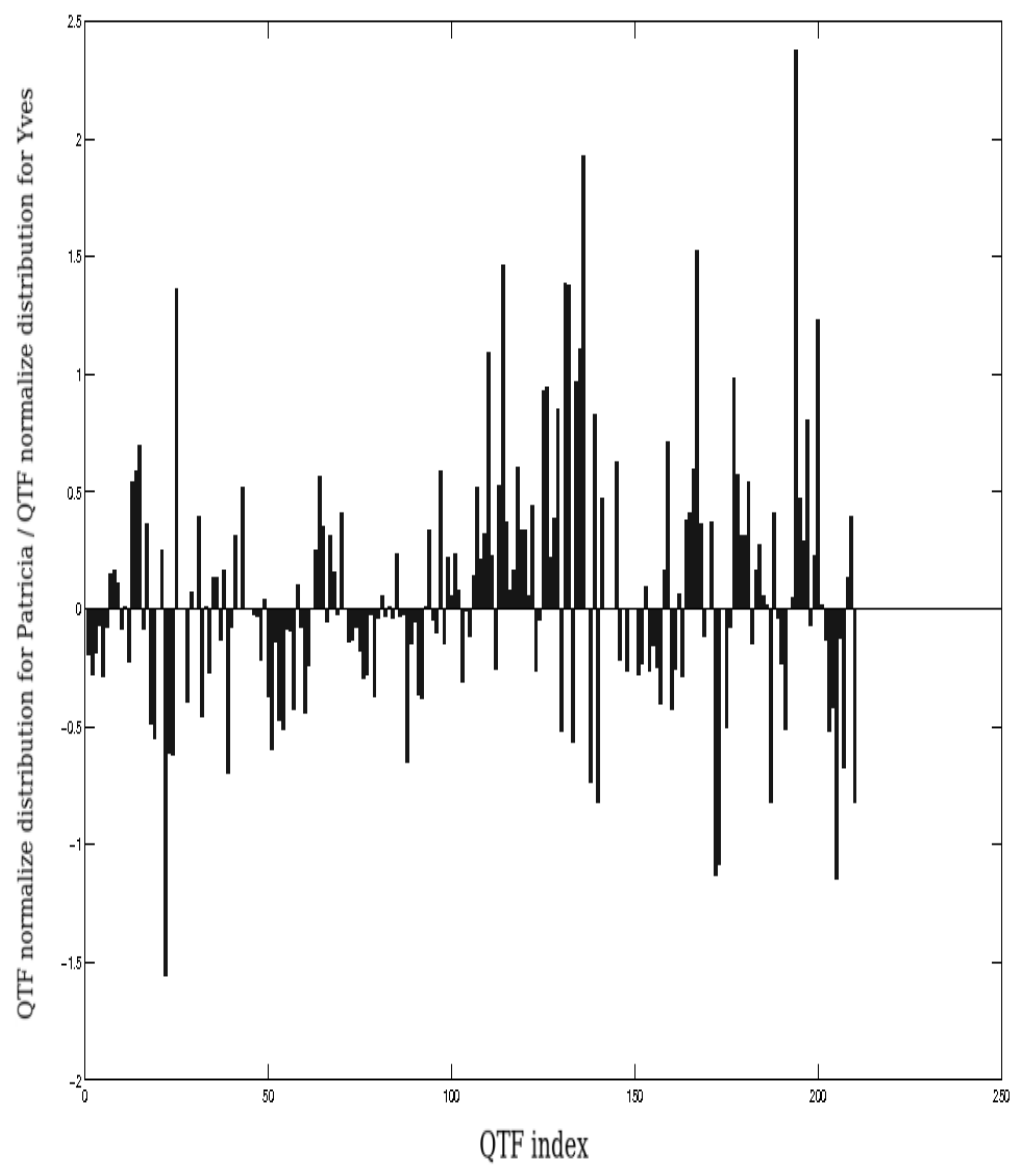

Fig. 5. This figure shows the logarithm (10) ratio between the QTF normalized distributions. The atomic relations and SB couples orders are the same than in previous figure. As we can see there are often large differences between at least half of the relations or SB couples. The MLP have learned with success each of these singularities distributions and then discriminats well speaker identity. Note that this figure represents only a part of the QTF processed information: it may exists another intra-frame discriminative information between the 15 parameters calculated on each frame, that is erased in this global histogram summing all training frames.

\section{Discussion and Conclusion}

A better optimisation could be obtained by combining different parameters for each subband. Moreover this preliminary iterative threshold research should be replaced by more global optimisation scheme. The total number of MLP weights is around $39000\left(=600^{*}(15+50)\right)$, which is smaller than usual parameters size. Nevertheless our approach generates the same order of errors than other experiments conducted on ESTER Phase 1 using classical MG HMM model on 
classical spectral parameters. Another major difference and originality of our qualitative approach remains its parcimonious and simple integer representation of each speaker in a very small integer subspace (50 speakers identity coded in $\left.[0: 13]^{15}\right)$.

A systematic falsification of each relation will give for each relation an idea of its amount of information for encoding speaker identity information. This should show if speakers are discriminated by particular relations, or if only the joint set of each relation encodes the speaker identity. Some experiments are currently testing our qualitative model on ESTER phase 2, which is a bigger database, with more speakers, and with published SRL and SVL scores with MG HMM models [17.

\section{Acknowledgements}

We thank Odile Papini for her information on logical atomic time representation. ADER for the CIFRE convention between DCN and LSIS UMR and USTV.

\section{References}

[1] Bimbot, F., Bonastre, J.-F., Fredouille, C., Gravier, G., Magrin-Chagnolleau, I., Meignier, S., Merlin, T., Ortega-Garcia, J., Petrovska, D., Reynolds, D.A.: A tutorial on text-independent speaker verification. EURASIP Journal on Applied Signal Processing 4, 430-451 (2004)

[2] Hayakawa, S., Takeda, K., Itakura, F.: Speaker Identification Using Harmonic Structure of LP-residual Spectrum. In: Bigün, J., Borgefors, G., Chollet, G. (eds.) AVBPA 1997. LNCS, vol. 1206, pp. 253-260. Springer, Heidelberg (1997)

[3] Greenberg, S., Arai, T., Grant, W.: The role of temporal dynamics in understanding spoken language. Dynamics of Speech Production and Perception Nato Advanced Studies Series, Life and Behavioural Sciences 374, 171-190 (2006)

[4] Fletcher, H.: The nature of speech and its interpretation. J. Franklin Inst. 193(6), 729-747 (1922)

[5] Allen, J.B.: How do humans process and recognise speech. IEEE Trans. on Speech and Signal Processing 2(4), 567-576 (1994)

[6] Glotin, H.: Elaboration and comparatives studies of robust adaptive multistream speech recognition using voicing and localisation cues. Inst. Nat. Polytech Grenoble \& EPF Lausanne IDIAP (2001)

[7] Morris, A., Hagen, A., Glotin, H., Bourlard, H.: Multi-stream adaptive evidence combination for noise robust ASR. int. journ. Speech Communication, special issue on noise robust ASR 17(34), 1-22 (2001)

[8] Glotin, H., Vergyri, D., Neti, C., Potamianos, G., Luettin, G.: Weighting schemes for audio-visual fusion in speech recognition. In: IEEE int. conf. Acoustics Speech \& Signal Process (ICASSP) Salt Lake City-USA (September 2001)

[9] Glotin, H.: When Allen J.B. meets Allen J.F.: Quantal Time-Frequency Dynamics for Robust Speech Features. Research Report LSIS 2006.001 Lab Systems and Information Sciences UMR CNRS (2006)

[10] Glotin, H.: Dominant speaker detection based on harmonicity for adaptive weighting in audio-visual cocktail party ASR. In: Adaptation methods in speech recognition ISCA Workshop September, Nice (2001) 
[11] Berthommier, F., Glotin, H.: A new SNR-feature mapping for robust multistream speech recognition. In: Proc. Int. Congress on Phonetic Sciences (ICPhS) Berkeley University Of California, Ed., San Francisco 1 of XIV August, pp. 711-715 (1999)

[12] Yumoto, E., Gould, W.J., Bear, T.: Harmonic to noise ratio as an index of the degree of hoarseness. The Acoustic Society of America 1971, 1544-1550 (1982)

[13] Allen, J.F.: An Interval-Based Representation of Temporal Knowledge. In: Proceedings of 7th IJCAI August, pp. 221-226 (1981)

[14] Allen, J.F.: Maintaining Knowledge About Temporal Intervals. Communications of the ACM 26(11), 832-843 (1983)

[15] Collobert, R., Bengio, S., Marihoz, J.: Torch: a modular machine learning software library. Laboratoire IDIAP IDIAP-RR 02-46 (2002)

[16] Gravier, G., Bonastre, J.F., Galliano, S., Geoffrois, E., Mc Tait, K., Choukri, K.: The ESTER evaluation campaign of Rich Transcription of French Broadcast News. In: Language Evaluation and Resources Conference (April 2004)

[17] Galliano, S., Geoffrois, E., Mostefa, D., Choukri, K., Bonastre, J.-F., Gravier, G.: The Ester Phase 2: Evaluation Campaign for the Rich Transcription of French Broadcast News. In: European Conf. on Speech Communication and Technology pp. 1149-1152 (2005) 